\title{
The Acute Effect of Aerobic Exercise on Serum Cortisol Levels of Athletes and Sedentary Individuals
}

\author{
Selahattin Koc \\ Correspondence: Selahattin Koc, Gaziantep University School of Physical Education and Sports, Turkey.
}

Received: November 21, 2018

doi:10.11114/jets.v6i12a.3901

\author{
Accepted: December 20, 2018 Online Published: December 24, 2018 \\ URL: https://doi.org/10.11114/jets.v6i12a.3901
}

\begin{abstract}
The purpose of this study was to investigate the effect of post-challenge acute values of serum cortisol levels in elite and sedentary athletes before aerobic exercise. This study included 22 elite middle-distance runners from various clubs around Turkey and 16 voluntary non-athlete men. The Cooper test was applied to study subjects. Heart rate values and blood samples of the subjects were taken before and after the Cooper test. The blood samples were analyzed at Central Biochemistry Laboratory of Şahinbey Research and Application Hospital of Gaziantep University. The results showed statistically significant difference between sedentary and athlete groups in comparison of BMI $(\mathrm{P}<0.05)$. A statistically significant difference was also found in pre-exercise cortisol and potassium levels and post-exercise lactate and potassium levels between both groups $(\mathrm{P}<0.05)$. No statistically significant difference was detected between groups in comparison of other blood parameters $(\mathrm{P}>0.05)$. Again, no statistically significant difference was found between pre-test and post-test values of pre and post-exercise Cortisol and Potassium levels of both groups $(\mathrm{P}>0.05)$.

Consequently, cortisol levels were same in both groups and were not affected by exercise. According to these results, it can be said that athletes' volume of air entering the lungs is higher than those who do not do sports, and the level of their anaerobic respiration is lower.
\end{abstract}

Keywords: aerobic, athlete, sedentary, exercise, cortisol.

\section{Introduction}

Athletes need a wide range and different levels of exercise to optimize their performance. The severity and range of exercises cause many hormonal changes in the bodies of athletes. Cortisol is one of these hormones that respond to training very quickly (Erdemir and Tüfekçioğlu, 2008). The rates of some hormones in the blood increase or decrease when compared in terms of exercise, training and resting values (Erdemir and Tüfekçioğlu, 2008). The main reason is the fact that the increases and decreases in exercise generally reflect adjustments in hormone amounts released by the endocrine gland. Also, it should be taken into account that they also can reflect changes in blood levels, metabolic turnovers and hemo-concentration effects (Erdemir and Tüfekçioğlu, 2008).

The effects of moderate exercise cause an increase in blood glucose by provoking the stress response to exercise, hence stimulating the secretion of insulin. In physically active individuals, the increases and decreases in blood glucose become irregular after glucose challenge during tolerance test. In moderate exercise, pyruvate and lactate concentration increases the density of plasma lactate twice due to an increase in the metabolic activities of skeletal muscles. The race between uric acid and tissue metabolism products with increased lactate for renal excretion causes an elevation in the density of serum urine. Decreased renal blood flow induces a mild increase in the density of serum creatine.

The impacts of severe exercise are an excessive version of the impact of moderate exercise. Severe exercise might cause hypoglycemia and increased glucose tolerance. Severe exercise can increase density of plasma lactate ten times; and decrease potassium concentration. Exercise creates a rapid increase in potassium, uric acid and muscle enzymes in intense races like marathons (Erdemir and Tüfekçioğlu, 2008).

Minerals also play unique roles in the body; potassium controls water balance in the body and Iron facilitates oxygen transport (http://sporis.net/spor-bilgi-bank.aspx, 2013). 


\subsection{Minerals}

Only few of the many elements in human body have observable biochemical or physiological functions. These elements can be divided in five main groups. The first group involves main constituents of body molecules such as carbon, hydrogen, oxygen, nitrogen and sulfur. The second group is composed of calcium, phosphorous, magnesium, sodium, potassium and chloride which are important minerals in terms of nutrition.

Group 5 elements are composed of elements with certainly known toxic effects such as lead and mercury. As many of the minerals contain poorly soluble salts and compounds, except for sodium and potassium, they cannot be absorbed in the body easily (http://www.saglik.im, 2013).

Minerals are very important for metabolic and physiological processes in the human body. In athletes, they are important for muscle contraction, cardiac rhythm, rhythm of nerve impulses, oxygen transportation, oxidative phosphorylation, immune functions, antioxidant activity, bone health and acid-base balance (Speich et al., 2001).

Minerals are present in all living cells and freely occur naturally in the earth and water. They are transported via the food chain by being absorbed in soil-grown plants and transferred to humans who consume those plants (Pehlivan, 2005). Experimental findings demonstrate that a deficiency of vitamins and minerals is rare in the general population when a balanced diet is applied (FNB, 2001). However, some individuals who expect high performance can take nutritional supplements (ADA, 2000). Vitamin and mineral supplements are suitable provided that scientific evidence support their safety and efficacy. This is important as physically active individuals might carry a high risk of nutritional deficiency, and vitamin and mineral supplements can be taken as a preventive measure as long as the recommended daily amount is not exceeded (ADA, 2001). The excessive intake of vitamins and minerals, except for folate, is a source of diet and supplement which disturbs foods and supplements (Lukaski, 2001). Weight etl, 1998; Tellford et al., 1992; Singh et a1., 992).

\subsection{Ions}

One of the most important ions in the cell is potassium (Guyton, 1991).

\subsection{Potassium}

Potassium has vital importance for nutrition as it helps protect the balance of water and minerals in the body. It is significant for the transportation of foods within cells, transmission of messages in nervous system as well as for heart and muscles. It eliminates alcohol, excess fluid, sugar and salt from the body (http://tr.mydearbody.com/mineraller, 2013).

Potassium deficiency is a mineral loss in the body due to malnutrition, diarrhea, diuretics, vomiting or sweating. Drinking excessive coffee and stress are among the causes of potassium deficiency. It causes disorders such as circulatory disorder, fatigue and tiredness associated with muscle weakness, lack of appetite, nausea and vomiting, mental confusion, headache and stomachache, cramps and cardiac arrhythmia. The most important causes of sports injuries are magnesium and potassium deficiencies (http://tr.mydearbody.com/mineraller, 2013).

Potassium has a stimulant effect when taken in small amounts and paralysis effect when taken in large amounts. It causes muscle contraction and increases tonus of heart (Kan, 2009).

In direct association with exercise, the concentration of potassium is released in muscle cells during exercise. Potassium is thought to trigger hyperkalemia with exercise. Hypokalemia caused by a shift from extracellular to intracellular space and is thought to be associated with potassium intake in the muscle following exercise as a consequence of stimulation of potassium ATPase catecholamine and sarcolemmal sodium without anaerobic metabolism or muscle ischemia. The resulting hypokalemia might cause increased intracellular acidosis with increased skeletal muscle blood flow. Exercise-induced hyperkalemia does not have any impact on athletes, and moreover, its effect can be even decreased (Welsh et al., 1999).

Exercise-induced hyperkalemia generally does not have any impacts on athletes. The physiological significance of exercise is not much in terms of potassium changes in healthy individuals; however, trainers and doctors should be aware of potential threats due to exercise (Warburton et al., 2002)

Normal muscle and neurological functions as well as normal blood pressure, brain and nervous functions depend on intracellular and extracellular regulations. A potassium supplement might cause a slight decrease in blood pressure (Olsen, 1979).

\subsection{Cortisol}

It is a corticosteroid hormone produced in the adrenal cortex within the adrenal gland and associated with the body's stress response. It increases blood pressure and sugar (wiki/Kortizol, 2013). 
Cortisol is measured in serum changes daily. The highest and lowest values are measured at night and in the morning a few hours after waking up, respectively. The underlying reasons of changes in serum cortisol levels are observed as unusual ACTH levels, clinical breakdown and physiological sources of stress (hypoglycemia, disease, fever, trauma, surgery, fear, pain, extreme cold or hot, physical constraint). Cortisol feeds back ACTH secretion by suppressing the secretion of corticotropin-releasing hormone (wiki/Kortizol, 2013).

When secreted under usual circumstances, cortisol shows impacts by restoring homeostasis after stress. Gluconeogenesis, the breakdown of lipids and proteins, leads to mobilization of extrahepatic amino-acids and ketone bodies. Thus, it acts as insulin antagonist and as a result, increases blood glucose levels and glycogenesis in the liver. It also elevates blood pressure. The continuous secretion of cortisol causes muscle abrasion, hyperglycemia and suppression of inflammation. Cortisol also slows down osteogenesis (wiki/Kortizol, 2013).

\subsection{Heart Rate}

Number of heart's total systolic contractions in one minute is called heart rate (HR) or, in other words, a pulse. Many factors such as age, gender, body position, food intake, body temperature, emotional moments, effect of exercise, environmental factors, etc. have an impact on heart rate (Nicolic and Ilic, 1992).

\subsection{Effect of Exercise}

The normal heart rate (HR) in healthy individuals is $60-100$ beats/min on average. The heart rate of middle-aged sedentary people can be 100 beats/ min while heart rate of very well trained athletes, particularly in those doing endurance training, can be 30-40 beats/min (Tiryaki Sönmez, G. 2002).

Maximum heart rate increases as the tempo of exercise increases. Energy needs and therefore, oxygen needs also increase. The heart starts to pump more blood to meet these needs, which consequently increases the heart rate. After a certain point, the heart reaches a level of tiredness and the number of pulses starts to decrease. This peak heart rate level is called the maximum heart rate (Tiryaki Sönmez, G. 2002).

Long-term heavy exercise leads to a range of biochemical changes. These changes are generally positive; however, they are potentially life-threating in some conditions, in particular, hyponatremia. Special attention should be paid as hyponatremia might potentially cause adverse outcomes as a consequence of long-term exercise (Welsh, et al. 1999).

\subsection{Exercise}

In the field of sport, physical fitness is an important factor as well as talent. Based on scientific data about different types of sports, physical activity is very influential on circulatory and respiratory systems during trainings and competitions. Oxygen needs vary according to the characteristics of the sport as well as the individual's vital capacity of the lungs and body structure. The chronic impact of training on respiration is increased vital capacity. The respiratory systems of trained athletes adapt to oxygen needs during training more rapidly (Sari et al., 1981).

Exercise is a systematical method that enables athletes to reach their highest sportive efficiency. Exercise increases the use of maximum oxygen in peripheral and central adaptations. How respiratory parameters are affected by exercise is assessed with mechanism characteristics, and sport is expected to increase these parameters in young people regardless of gender (Sari et al., 1981). Exercise is defined as body movements for increasing strength and endurance, improve functions and restore disorders, if any, in humans (Koç, 1997).

\subsection{Pulse}

A pulse is a counting heart rate. In other words, it indicates how many times a heart beats per minute. When contracted, the heart pumps blood to vessels (each time $60-70 \mathrm{ml}$ on average) (http://tip.sdu.edu.tr)

Heart rate per minute is 60 to 100 in normal adults. Heart rate can increase when one moves as well as in cases of diseases, injuries and emotional situations (http://tip.sdu.edu.tr).

The resting heart rate of athletes with increased circulation is around 40, which is normal. Arteries, over which the pulse is taken, are the ones closer to surface. The most frequently used pulses are radial and ulnar pulses on the wrist and brachial pulse on the inner side of elbow. The index finger, middle finger and ring finger are pressed slightly on pulse and after counting for 15 seconds, number is multiplied by four to get a pulse per minute. In cardiac patients, it is beneficial to count for one minute. Heart rate above 100 per minute and below 60 per minute are called tachycardia and bradycardia, respectively (http://tip.sdu.edu.tr).

\section{Method}

This study aimed to examine the impact of hormones, minerals and lactate on elite athletes running middle distance from various sports clubs in Turkey. As data collection tool, pulse values of the groups of elite middle-distance runners and non-athletes were recorded prior to exercise and after an exercise of a $1000 \mathrm{~m}$ run, blood samples were taken, and selected 
parameters were analyzed on 22 athletes and 16 non-athletes at Central Biochemistry Laboratory of Şahinbey Research and Application Hospital of Gaziantep University. As a consequence of analyses, the mean body mass index was at expected values of healthy individuals in athletes while statistically significant and higher in non-athletes. Cortisol levels were the same in both groups and were not affected by exercise. In both groups, ACTH hormone levels were the same and ACTH values doubled after exercise. While pre-exercise lactic acid levels were similar in both groups, post-exercise lactic acid levels increased twice in non-athletes compared to athletes with a statistically significant difference. According to these results, it is understood that athletes' volume of air entering the lungs is higher than those who do not do sports, and the level of their anaerobic respiration is lower. The pre and post exercise serum free iron level was lower in athletes than non-athletes; however, the difference was not statistically significant $(\mathrm{p}>0.05)$. The pre and post exercise iron binding capacity of athletes were found statistically and significantly higher than non-athletes. This study suggests that sports increases iron binding capacity, and therefore, serum transferrin level also gets higher. It was understood in this study that being trained while doing sports did not affect serum ion values.

\subsection{Study Population and Sample}

This study aimed to examine the impact of hormones, minerals and lactate on elite athletes running middle distance from various sports clubs in Turkey.

\subsection{Data Collection Tools}

As data collection tool, the pulse values of the groups of elite middle-distance runners and non-athletes were recorded prior to exercise and after an exercise of a $1000 \mathrm{~m}$ run, blood samples were taken, and selected parameters were analyzed on 22 athletes and 16 non-athletes at Central Biochemistry Laboratory of Şahinbey Research and Application Hospital of Gaziantep University.

\section{Results}

41 patients were included in this study. 16 of them formed the non-athlete group and 25 formed the athlete group (Table 1).

Table 1. Distribution of non-athlete control group and athlete group in the study.

\begin{tabular}{lrl}
\hline \multicolumn{1}{c}{ Groups } & Frequency & Percent \\
\hline Sedentary & 16 & 39.0 \\
Athlete & 25 & 61.0 \\
Total & 41 & 100.0 \\
\hline
\end{tabular}

Table 2 suggests that the mean age of individuals forming these groups is close. Again as seen in Table 6, it is understood that the body mass indices are significantly lower and therefore, percentage of muscle is higher and percentage of fat is lower in athletes (Table 6).

Table 2. Age and BMI (body mass index) values of non-athlete and athlete groups.

\begin{tabular}{lllccc}
\hline & Group & N & Mean & Std. Deviation & P \\
\hline \multirow{2}{*}{ Age } & Sedentary & 16 & 22.44 & 1.263 & 0.295 \\
& Athlete & 25 & 21.52 & 3.293 & \\
\multirow{2}{*}{ BMI } & Sedentary & 16 & 26.8484 & 1.84557 & $0.001^{*}$ \\
& Athlete & 25 & 22.1092 & 2.10310 & \\
\hline
\end{tabular}

The analysis values of blood parameters in athletes and non-athletes are shown in Tables 3,4 and 5. When these values were examined, it was seen that lactic acid level significantly increased in non-athletes compared to athletes. The increase was approximately $100 \%$ (Table 3). Another significant increase was seen in blood ACTH levels and accordingly, post-exercise ACTH levels in athletes and non-athletes specifically increased compared to pre-exercise levels (Table 3). When these values were compared to intragroup values, the same increase was found statistically significant (Tables 4 and 5).

While pre-exercise and post-exercise blood sodium values remained the same in non-athletes, a relative decrease was seen in athletes (Table 3). These values show similarity in both intergroup and intragroup analyses and are statistically non-significant (Tables 3, 4 and 5). 
Table 3. Analysis of blood parameters in athletes and non-athletes

\begin{tabular}{|c|c|c|c|c|c|}
\hline & Group & $\mathbf{N}$ & Mean & Std. Deviation & $\mathbf{P}$ \\
\hline \multirow{3}{*}{ Cortisol $(\mu \mathrm{g} / \mathrm{dL}) *$ pre-e } & Sedentary & 16 & 13.18 & 3.498 & \multirow{3}{*}{0.365} \\
\hline & Athlete & 24 & 11.76 & 5.450 & \\
\hline & Sedentary & 16 & 13.619 & 3.1143 & \\
\hline \multirow[t]{2}{*}{ Cortisol $(\mu \mathrm{g} / \mathrm{dL}) *$ post-e } & Athlete & 25 & 14.612 & 5.7061 & \multirow[t]{2}{*}{0.477} \\
\hline & Athlete & 25 & 135.64 & 25.837 & \\
\hline \multirow{3}{*}{ Potassium (mmol/L) pre-e } & Sedentary & 16 & 4.6106 & .33840 & \multirow{3}{*}{$0.037 *$} \\
\hline & Athlete & 25 & 4.3920 & .30202 & \\
\hline & Sedentary & 16 & 4.750 & .5247 & \\
\hline \multirow[t]{2}{*}{ Potassium (mmol/L) post-e } & Athlete & 25 & 4.102 & .3218 & \multirow[t]{2}{*}{$0.001^{*}$} \\
\hline & Athlete & 25 & 106.24 & 2.204 & \\
\hline
\end{tabular}

Abbreviations have the following meanings: *pre-e= pre-exercise, $* *$ post-e= post-exercise.

Table 4. Results of analysis values of intragroup blood parameters before and after sports in non-athlete group

\begin{tabular}{|c|c|c|c|c|c|}
\hline & & Mean & $\mathbf{N}$ & Std. Deviation & $\mathbf{P}$ \\
\hline \multirow{4}{*}{ 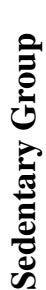 } & Cortisol pre-e & 13.18 & 16 & 3.498 & \\
\hline & Cortisol post-e & 13.619 & 16 & 3.1143 & 0.601 \\
\hline & Potassium pre-e & 4.6106 & 16 & .33840 & \\
\hline & Potassium post-e & 4.750 & 16 & .5247 & 0.342 \\
\hline
\end{tabular}

Table 5. The athlete group's results of analysis values of intragroup blood parameters before and after sports

\begin{tabular}{lllccc}
\hline & Mean & N & Std. Deviation & P \\
\hline \multirow{2}{*}{ Cortisol pre-e } & Cortisol post-e & 11.76 & 24 & 5.450 & $0.012^{*}$ \\
& 15.188 & 24 & 5.0334 & $0.001 *$ \\
\hline
\end{tabular}

As a result, among blood parameters, lactic acid and free iron levels in blood were higher in non-athletes compared to athletes, and an increase was seen in both parameters after exercise. ACTH levels in both athletes and non-athletes increased approximately twice after exercise, and the increases were similar (Tables 3, 4 and 5). According to these results, no difference was observed in electrolyte levels and essential macro element levels of both groups (Tables 4,5 and 6).

Table 6. Results of pulse measurement values after exercise

\begin{tabular}{llrlrl}
\hline & \multicolumn{1}{c}{ Group } & N & \multicolumn{1}{c}{ Mean } & Std. Deviation & P \\
\hline Pulse 1 & Athlete & 25 & 164.4800 & 15.63415 & 0.001 \\
& Sedentary & 16 & 199.7500 & 6.72805 & 0.001 \\
Pulse 2 & Athlete & 25 & 125.36 & 14.871 & 12.263 \\
\multirow{2}{*}{ Pulse 3 } & Sedentary & 16 & 170.88 & 14.838 & 0.001 \\
& Athlete & 25 & 103.92 & 17.025 & \\
\hline
\end{tabular}




\section{Discussion and Conclusion}

Cellular metabolic functions are used more efficiently via bodily mobility. The unique functional properties of cells distributed across various organs also regulate metabolic functions according to the functions of these cells. Therefore, vital activities of cellular organisms in organs and tissues constitute all functions assumed and performed by these cells. For that reason, cells are composed of various organelles in higher life forms and organelles of the cells perform different functions at different levels according to the organ and tissue they are contained in. As individuals maintain their normal bodily mobility, their cells in organs and tissues perform functions in an efficient manner by using catabolism and anabolism actively through nutrients.

Cells in organs and tissues are also surrounded by interstitial fluid. This interstitial fluid ensures exchange of metabolites between cells, maintains polarity of cell membranes and enables cell response to stimulation as well as cellular transmission. This interstitial fluid is therefore an electrolyte environment and plays an important role in ensuring intracellular electrolyte balance (Ganong, 1979). As humans and animals have a closed circulatory system, extracellular fluid (ECF) has two important elements. Among these extracellular fluids, one of them is interstitial fluid surrounding the cells and the other is blood plasma in circulation. Plasma and red blood cells in blood circulation make up total blood volume. Interstitial fluids are fluids outside blood circulation. Also, intracellular fluids constitute a significant part of total body fluids. While $70 \%$ of total body fluid is intracellular in an adult person, the remaining amount above 30\% is extracellular (Ganong, 1979). Intracellular tonus (turgidity), osmolarity, intercellular diffusion, metabolite exchange, buffer solution between intracellular and extracellular compartments, potential of membrane by polarity between intracellular and extracellular compartments depend on electrolyte balance between intracellular and extracellular compartments. This balance is also about balancing electrolytes between interstitial fluid and extracellular fluid and circulatory blood fluid. The regulation of many physiological functions in cells and organs and, in general, organism is associated with a internal balance of organism (Ganong, 1979).

Nutrition is very significant in preserving the concentration of cations and anions in the formation of electrolyte environment and buffer solution. Restoring anions and cations lost during vital processes is associated with balanced nutrition. Minerals and trace elements are not only important for organismal electrolyte balance but also allows efficient functioning of metabolic mechanisms thanks to their presence in protein and enzyme structures.

$\mathrm{Na}^{+}$ions in electrolyte structure is absorbed in large intestine and jejunum, duodenum and ileum parts of small intestine. Hydrogen ions $\left(\mathrm{H}^{+}\right)$and bicarbonate acids $\left(\mathrm{HCO}_{3}\right)$ are absorbed partially in the jejunum, absorbed moderately in the duodenum and ileum, and absorbed and released in the large intestine (Ganong, 1979).

Hormones synthesized by the adrenal cortex are cholesterol derivatives. Secreted corticosteroid hormones are derivatives of $\mathrm{C}_{21}$, and as mentioned before, those with mineral corticoid activity regulate $\mathrm{Na}^{+}$and $\mathrm{K}^{+}$excretion while those with glucocorticoid activity adjust glucose and protein metabolisms. Primary $\mathrm{C}_{21}$ steroids synthesized in adrenal tissue and released at physiological activity level are aldosterone, cortisol and corticosterone. Cortisol, a glucocorticoid, is mostly bound to transcortin in circulation which is also known as corticosteroid-binding $\alpha$-globulin (CBG). Cortisol is loosely bound to albumin. Similarly to cortisol, corticosterone is bound to protein at lower amounts. Therefore, a half-life of circulating corticosterone (50 minutes) is much shorter than cortisol (60 to 90 minutes). Protein-bound cortisol and corticosterone are physiologically non-functional, and free cortisol and corticosterone are slightly excreted in urine. The balance between free cortisol and bound cortisol depends on the amount in tissue and ACTH secretions (Ganong, 1979).

The CBG protein is synthesized in the liver and its synthesis increases in the presence of estrogen. Level of CBG in blood increases during pregnancy and decreases in liver cirrhosis, kidney disorder (nephrosis) and multiple myeloma. When CBG level increases, cortisol in blood transform into more CBG-bound form and in the meantime, free cortisol decreases temporarily and ACTH secretion into blood increases with these decrease. Elevated ACTH in the blood increases cortisol release into blood until balance is restored, and thus ensures a normal level of cortisol in the blood. During this balancing process, the level of bound cortisol is kept elevated to ensure a normal level of ACTH secretion. Because high levels of glucocorticoids (cortisol) in the blood suppress ACTH secretion, therefore, the suppression level of the pituitary gland is in direct proportion with the level of free glucocorticoids in the blood. ${ }^{(45)}$ This suppression is rapid and long-term as the level of cortisol decreases slowly. The elevation of ACTH level in the blood triggers glucocorticoid release from the adrenal gland, and glucocorticoids maintain a normal level of ACTH in the blood by controlling its secretion with a feedback mechanism. As sudden discontinuation of steroid treatment applied when this balance is disturbed might lead to many metabolic complications, cortisol treatment is ended through an adjustment of long-term dose reduction ${ }^{(16)}$.

As the level of free glucocorticoids in plasma is very low (approximately $0.5 \mu \mathrm{g} / \mathrm{dl}$ ), the suppression of the pituitary gland is also low under normal conditions, and decreased cortisol level triggers ACTH secretion in case of stress. 
Exhausting or stress-causing stimulation generally activates sympathoadrenal medullary system while triggering ACTH secretion. ${ }^{(45)}$ Sympathoadrenal medullary system is the first system that responds to early phase of exhausting stimulation and triggers the release of noradrenaline and adrenaline which are neuroendocrine hormones. In the meantime, catecholamine response to stress also increases (Gabrilovic, Dronjak, 2006). Stimulation of sympathoadrenal medullary system occurs more distinctly with psychological stress rather than physical stress (Gabrilovic, Dronjak, 2006). The presence of circulating glucocorticoids with exhausting physical movements might be associated with ensuring the vessels' sensitivity to catecholamines (Ganong, 1979). As mentioned earlier, the activity of glucocorticoids is required for catecholamines in fatty acid metabolism and transportation in blood. Again as underlined before, free fatty acids play a significant role to help elevated glucocorticoid levels in the blood ensure body resistance against stress as energy sources in case of sudden and urgent energy requirements. Glucocorticoids increase the catabolism of proteins and accelerate hepatic glycogenolysis (breakdown of glycogen) and gluconeogenesis (synthesis of glucose). ${ }^{(45,46)}$ The function of glucose 6-phosphate increases and glucose level in the blood elevates (Ganong, 1979; Devlin, 2006). In addition to glucocorticoids' positive effects on glycogenolysis and gluconeogenesis, their anti-insulin function in peripheral tissues has adverse effects on the control of sugar levels in diabetic patients (Ganong, 1979; Devlin, 2006). The two impacts of glucocorticoids are that they do not affect the brain and heart tissues and they regularly provide glucose to these essential organs (Ganong, 1979). Glucocorticoids increase plasma fat content and the formation of ketone bodies in Type 2 diabetes; however, in normal healthy individuals, healthy living conditions are maintained to keep blood glucose level within normal ranges through regulation of insulin secretion, synthesis of fats from excess glucose in fatty tissue, and glycogenesis and lipogenesis in muscle and liver tissues as blood glucose level increases (Ganong, 1979; Devlin, 2006). Hormonal release by the adrenal cortex plays no role in the development of ketogenesis. In adrenal insufficiency, an individual can maintain a normal life as long as daily calorie need is met; however, patients with this disease can develop potentially fatal hypoglycemia in case of fasting or starvation (Ganong, 1979).

As seen in Table 6, it is understood that body mass indices are significantly lower and therefore, percentage of muscle is higher and percentage of fat is lower in athletes. The analysis values of blood parameters in athletes and non-athletes are shown in Tables 3, 4 and 5.

\section{References}

American Dietetic Association (2000). Position of the American Dietetic Association, Dietitians of canada, and the American College of Sport Medicine: nutrition and athletic performance. J Am Diet Assoc, 100, 1543. https://doi.org/10.1016/S0002-8223(00)00428-4

Devlin, T. M. (2006). Carbohydrate metabolism I. Major metabolic pathways and their control. Textbook of Biochemisytry with Clinical Correlation. 6th Ed. Wiley-Liss, Hoboken, NJ. 618-633.

Devlin, T. M. (2006). Lipid metabolism I: Synthesis, storage, and utilization of fatty acids and triacyl glycerols. Textbook of Biochemisytry with Clinical Correlation. 6th Ed. Wiley-Liss, Hoboken, NJ. 661-693.

Erdemir, İ., \& Tüfekçioğlu, E. (2008). Comparison of Some Physical and Physiological Parameters Affecting Cortisol Circadian Rhythm, Ballkesir University Journal of Institute of Social Sciences, 11(20), 1-10, Balıkesir.

Food and Nutrition Board (2001). Institute of Medicine. Dietary reference intakes for vitamin A, vitamin K, arsenic, boron, chromium, copper, iodine, iron, manganese,. Washington, DC: National Academy Pres.

Gabrilovic L., \& Dronjak, S. (2006). Sympatho-adrenomedullary system responses to various chronic stress situations. Jugoslav Med Biochem, 24, 11-15.

Ganong, W. F. (1979). Digestion and absorption. Review of Medical Physiology. 9th Ed. LANGE Medical Publications, Los Altos, CA. 362-370.

Ganong, W. F. (1979). Physiologic principles. Review of Medical Physiology. 9th Ed. Lange Medical Publications, Los Altos, CA. 1-20.

Ganong, W. F. (1979). The adrenal medulla and adrenal cortex, Review of Medical Physiology. 9th Ed. Lange Medical Publications, Los Altos, CA. 1979, 277-300.

Guyton, A. C. (1991). Medical Physiology, Missipi University Medical Center Department of Physiology and Biophysics, 7, 424-425.

Kan, Ö. (2009). The Effect of 12-Week Anaerobic Training Program on Blood Lactate and Electrolyte Levels of 14-16 Year-Old Male Taekwondociler, Selçuk University Institute of Health Sciences, Coaching Department, Unpublished Master Thesis, 14-15, Konya.

Koç, S. (1997). The effect of different types of sports on hematological biochemical and some respiratory parameters in young girls, F.Ü. Faculty of Arts and Sciences Department of Physical Education and Sports, Master Thesis, 
Elazı̆̆.

Lukaski, H. C. (2001). Magnesium, zinc and chromium nutrition and athletic performance. Can. J. Appl. Physiol., 26(suppl), 13. https://doi.org/10.1139/h2001-038

Nicolic, Z., \& Ilic, N. (1992). Maximsal Oxygen Uptake in Trained And Untrained 15 Year-Old Boys, PMID:1600452, Mar; 26(1), 36-8. http://www.saglik.im/ (Date Of Access, 11.01.2013).

Olsen, R. E. (1979). Vitamin and Modrn Nutrition in Health and Disease. 9th ed.

Pehlivan, A. (2005). Nutrition in Sports, Morpa Cultural Publications, 107-116, İstanbul.

Sari, H., Terzioğlu, M., \& Erdoğan, F. (1981). Comparison of respiratory-circulation parameters in resting exercise and relaxation of athletes in different sports branches and sedentary people. Sport hek. J., 16(4), 121-133.

Singh, A., Moses, F. M., \& Deuster, P. A. (1992). Chronic multivitamin-mineral supplementation does not enhance physical performance. Med Sci Sports Exerc; 24, 726. https://doi.org/10.1249/00005768-199206000-00017

Speich, M., Pineau, A., \& Ballereau, F. (2001). Minerals, trace elements and related biological variables in athletes and during physical activity. Clin Chim Acta 312, 1-11. https://doi.org/10.1016/S0009-8981(01)00598-8

Tellford, R., Catchpole, E., Deakin, V., Hahn, A., Plank, A. W. (1992). The effect of 7 to 8 months of vitamin/mineral supplementation on athletic performance. Int Sports Nutr., 2, 135.

Tellford, R., Catchpole, E., Deakin, V., Mcleay, A., Plank, A. W. (1992). The effect of 7 to 8 months of vitamin/mineral supplementation on the vitamin and mineral status of athletes. Int J Sports Nutr., 2, 123.

Tiryaki, S. G. (2002). Exercise and Sport Physiology, 162-164-176-177, Bolu.

Warburton, D. E. R., Welsh, R. C., Haykowsky, M. J., Taylor, D. A., \& Humen, D. P. (2002). Biochemical changes as a result of prolonged strenuous exercise, Br. J. Sports Med., 36, 301-303. https://doi.org/10.1136/bjsm.36.4.301

Weight, L. M., Noakes, T. D., Labadarios, D., Graves, J., Jacobs, P., \& Berman, P. A. (1988). Vitamin and mineral status of trained athletes including the effects of supple mentation. Am. J. Clin. Nutr., 47, 186. https://doi.org/10.1093/ajcn/47.2.186

Welsh, R., Warburton, D. E. R., \& Haykowksy, M. J. (1999). Hematological responce to the half ironman triathion Med Sci Sport Exerc., 31, 63.

\section{Copyrights}

Copyright for this article is retained by the author(s), with first publication rights granted to the journal.

This is an open-access article distributed under the terms and conditions of the Creative Commons Attribution license which permits unrestricted use, distribution, and reproduction in any medium, provided the original work is properly cited. 